\title{
MARKOV OPERATORS AND QUASI-STONIAN SPACES
}

\author{
ROBERT E. ATALLA
}

\begin{abstract}
Let $X$ be a quasi-stonian space, and let $T$ be a $\sigma$-additive Markov operator on $C(X)$. Ando proved that if all $T$-invariant probabilities are $\sigma$-additive, then $T$ is strongly ergodic (and the space of fixed points is finite-dimensional). We prove that if the set of $\sigma$-additive $T$-invariant probabilities is weak-* dense in the set of all $T$-invariant probabilities, then $T$ is strongly ergodic. This result is easy in case $X$ is hyperstonian. Our method of proof is to use an idea of Gordon to "hyperstonify" part of our quasi-stonian space.
\end{abstract}

1. Introduction. A compact space is quasi-stonian if any of the following equivalent conditions hold: (1) if $A$ is an open $F_{\sigma}$ set, then closure $(A)$ is clopen ( $=$ closed and open), (2) $X$ is totally disconnected, and the algebra of clopen sets is $\sigma$-complete, (3) $C(X)$ is conditionally $\sigma$-complete. A Borel measure $m$ on $X$ is o-additive if $f_{n} \in C(X), f_{1}>f_{2}>\cdots>0$, and $\wedge f_{n}=0$ imply $|m|\left(f_{n}\right) \rightarrow 0$. The operator $T$ on $C(X)$ is $\sigma$-additive if $\wedge T f_{n}=0$ whenever $f_{n}$ is as above [An, Section 2]. Let $\Sigma(X)$ be the Banach space of $\sigma$-additive elements of the dual space $C(X)^{*}$. If $T$ is a Markov operator on $C(X)$, i.e., $T \geqslant 0$ and $T e=e$, where $e$ is the unit function, let $F(T)=\{f$ in $C(X): T f=f\}, F\left(T^{*}\right)=\left\{m\right.$ in $\left.C(X)^{*}: T^{*} m=m\right\}$ (where $T^{*}=$ adjoint of $T$ ), and $P\left(T^{*}\right)$ be the set of probability measures in $F\left(T^{*}\right)$. Write $T_{n}=(1 / n)\left(I+\cdots+T^{n-1}\right)$. $T$ is called strongly ergodic if for each $f$ in $C(X), \lim T_{n} f$ exists in the Banach space $C(X)$. Our main result is

1.1. TheOREM. If $X$ is quasi-stonian, $T$ a $\sigma$-additive Markov operator on $C(X)$, and $P\left(T^{*}\right) \cap \Sigma(X)$ is weak-* dense in $P\left(T^{*}\right)$, then $T$ is strongly ergodic.

1.2. Corollary [An, Theorem 2]. If $X$ is quasi-stonian and $T$ a $\sigma$-additive Markov operator on $C(X)$, the following are equivalent:

(a) $F\left(T^{*}\right) \subset \Sigma(X)$,

(b) $T$ is strongly ergodic, and $\operatorname{dim}(F(T))=\operatorname{dim}\left(F\left(T^{*}\right)\right)<\infty$.

We note that Sato has extended Ando's theorem to include certain non-Markov positive operators [Sa, Theorem 1]. Sato's theorem is not in any obvious way a corollary to 1.1 .

$\S 2$ is devoted to some general ergodic theory, and $\$ 3$ to the proofs of 1.1 and 1.2 .

1.3. Some concepts. A compact space is stonian if any of the following equivalent conditions hold: (1) if $A$ is open, then $\operatorname{closure}(A)$ is open, (2) $X$ is totally

Received by the editors October 30, 1979 and, in revised form, November 20, 1979 and September 29, 1980.

1980 Mathematics Subject Classification. Primary 47A35; Secondary 54G05.

() 1981 American Mathematical Society 0002-9939/81/0000-0370/\$02.50 
disconnected, and the Boolean algebra of clopen sets is complete, (3) $C(X)$ is a conditionally complete lattice.

To define hyperstonian spaces, we need the concept of normal measure, i.e., a measure $m$ which satisfies any of the equivalent conditions: (1) $m$ is order-continuous, i.e., if $\left\{f_{a}\right\}$ is a directed family in $C(X)$ and $f=\sup _{a} f_{a}$, then $\int f d m=$ $\lim _{a} \int f_{a} d m$, (2) if $F$ is a closed nowhere dense set, then $m(F)=O$ [Sch, p. 149, exercise 24]. Let $\Sigma_{0}=\Sigma_{0}(X)$ be the Banach space of all normal measures.

A compact $X$ is hyperstonian if any of the following equivalent conditions hold: (1) $C(X)=\left(\Sigma_{0}\right)^{*}$, i.e., $C(X)$ is the Banach space dual of $\Sigma_{0}$ (where $\Sigma_{0}$ has its norm topology), (2) $X$ is stonian and $\Sigma_{0}$ is weak-* dense in $C(X)^{*},(3) X$ is stonian and the union of the support sets of measures in $\Sigma_{0}$ is dense in $X$ [Sch, pp. 121-122].

Clearly, a normal measure is $\sigma$-additive, as we have defined it above. In the course of proving Theorem 1.1, we shall introduce a new topology under which, as we shall prove, the $\sigma$-additive measures become normal measures.

2. Criteria for ergodicity. If $R$ is an operator on the Banach space $B$, let $R^{*}=\operatorname{adjoint}(R), F(R)=$ fixed points of $R$, and $F\left(R^{*}\right)=$ fixed points of $R^{*}$. Write $R_{n}=(1 / n)\left(I+\cdots+R^{n-1}\right) . R$ is strongly ergodic if $\lim R_{n} x$ exists for each $x$ in $B$. If $R$ is a contraction, then $R$ is strongly ergodic iff $F(R)$ separates $F\left(R^{*}\right)$, i.e., $F\left(R^{*}\right) \cap F(R)^{\perp}=(0)\left[S_{2}\right]$. More generally, the separation criterion is valid if $R$ satisfies

$$
\left\|R_{n}\right\|<M(n>1), \text { and } \lim \inf n^{-1}\left\|R^{n}\right\|=0
$$

[L⿸广 $\mathbf{L}_{1}$, p. 123]. Note that if (1) holds for $R$, then it holds for $R^{*}, R^{* *}$, etc.

2.1. Proposition. If $R$ satisfies (1), the following are equivalent:

(a) both $R$ and $R^{*}$ are strongly ergodic,

(b) $F\left(R^{* *}\right)=\sigma\left(B^{* *}, B^{*}\right)$-closure of $F(R)$,

(c) norm-closure $\left((I-R)^{*}\left(B^{*}\right)\right)=$ weak-* closure $\left((I-R)^{*}\left(B^{*}\right)\right)$.

Proof. First note that in general weak-* $\operatorname{closure}\left((I-R)^{*}\left(B^{*}\right)\right)=F(R)^{\perp}$, and $\sigma\left(B^{* *}, B^{*}\right)$-closure $(F(R))=F(R)^{\perp \perp}$.

(c) implies (b). In general, $F\left(R^{* *}\right)=$ norm-closure of $\left.(I-R)^{*}\left(B^{*}\right)\right\}^{\perp}$, so if norm-closure $\left((I-R)^{*}\left(B^{*}\right)\right)=F(R)^{\perp}$, then $F\left(R^{* *}\right)=F(R)^{\perp \perp}$.

(b) implies (c). If (c) fails, then there exists $m$ in $F(R)^{\perp} \backslash$ norm-closure of $(I-R)^{*}\left(B^{*}\right)$. By Hahn-Banach there exists $F$ in $B^{* *}$ with $F(m)=1$ and $F\left(n-R^{*} n\right)=0$ for all $n$ in $B^{*}$. Since $F$ is in $F\left(R^{* *}\right) \backslash F(R)^{\perp \perp}$, (b) fails.

(c) implies (a). In general, norm-closure $\left((I-R)^{*}\left(B^{*}\right)\right) \cap F\left(R^{*}\right)=(0)$, so if (c) holds, then $F(R)^{\perp} \cap F\left(R^{*}\right)=(0)$, and hence $R$ is strongly ergodic, by the separation theorem. Hence there exists a projection $S$ on $F(R)$ with kernel closure $((I-R)(B))$, and then $S^{*}$ yields the decomposition $B^{*}=F\left(R^{*}\right) \oplus F(R)^{\perp}$. But then (c) implies $B^{*}=F\left(R^{*}\right) \oplus$ norm-closure $\left((I-R)^{*}\left(B^{*}\right)\right)$, and this implies (by direct computation of averages) that $R^{*}$ is strongly ergodic.

(a) implies (c). If $R$ is strongly ergodic, then (as noted above) $B^{*}=F\left(R^{*}\right) \oplus$ $F(R)^{\perp}$. If $R^{*}$ is strongly ergodic, then $B^{*}=F\left(R^{*}\right) \oplus$ norm-closure $\left((I-R)^{*}\left(B^{*}\right)\right)$. Since norm-closure $\left((I-R)^{*}\left(B^{*}\right)\right) \subset F(R)^{\perp}$, we have equality. 
In the next result, $X$ will be a hyperstonian space, as defined in $\S 1.3$, with normal measures $\Sigma_{0}$. Note that if $R$ is a $\sigma$-additive Markov operator, then $R^{*}\left(\Sigma_{0}\right) \subset \Sigma_{0}$. Let $S=R^{*} \mid \Sigma_{0}$. Then $S$ is norm continuous on $\Sigma_{0}$, and $S^{*}=R, S^{* *}=R^{*}$.

2.2. Proposition. Let $R$ be a $\sigma$-additive Markov operator on $C(X)$, where $X$ is hyperstonian. Then $R$ is strongly ergodic iff

$$
F\left(R^{*}\right)=\text { weak-* } \operatorname{closure}\left(F\left(R^{*}\right) \cap \Sigma_{0}(X)\right) .
$$

Proof. Necessity. If $R$ is strongly ergodic, then there exists a projection $Q$ such that $R_{n} f \rightarrow Q f(f$ in $C(X)) . Q$ is easily seen to be $\sigma$-additive, and hence $Q^{*}\left(\Sigma_{0}(X)\right) \subset \Sigma_{0}(X) . F\left(Q^{*}\right)$ is weak-* closed and $Q^{*}$ is weak-* continuous, so $F\left(R^{*}\right)=F\left(Q^{*}\right)=Q^{*}\left(C(X)^{*}\right)=$ weak-* $\operatorname{closure}\left(Q^{*}\left(\Sigma_{0}(X)\right)\right)$. Since $Q^{*}\left(\Sigma_{0}(X)\right) \subset$ $\Sigma_{0}(X)$ and $Q^{*}\left(\Sigma_{0}(X)\right) \subset F\left(R^{*}\right)$, we have $F\left(R^{*}\right) \subset$ weak-* closure $\left(\Sigma_{0}(X) \cap F\left(R^{*}\right)\right)$.

Sufficiency. Put $S=R^{*} \mid \Sigma_{0}(X)$. Then, since $R=S^{*}, R^{*}=S^{* *}, C(X)=\left(\Sigma_{0}\right)^{*}$, and $C(X)^{*}=\left(\Sigma_{0}\right)^{* *}$, the conclusion reads $F\left(S^{* *}\right)=\sigma\left(\Sigma_{0}^{* *}, \Sigma_{0}^{*}\right)$-closure of $F(S)$. By “(b) implies (a)" of Proposition 2.1, it follows that $S$ and $S^{*}=R$ are strongly ergodic.

\section{Proofs.}

3.1. Proof of Theorem 1.1. First we need some topological observations. A closed set $S$ is a $P$-set if the intersection of a countable number of neighborhoods of $S$ is again a neighborhood of $S$, or, equivalently, if $A$ is an open $F_{\sigma}$ which is disjoint from $S$, then $\operatorname{closure}(A)$ is disjoint from $S$. By $\left[\mathbf{L}_{2}\right.$, Lemma 1$]$, if $X$ is quasi-stonian and $m$ a $\sigma$-additive regular Borel probability, then $\sup (m)$ is a $P$-set. (By $\sup (m)$ we mean its support, i.e., the smallest closed set $S$ such that $m(S)=m(X)=1$.) (To make the paper more self-contained, we sketch a proof. Let $\boldsymbol{m}$ be $\sigma$-additive, $S=\sup (m)$, and $A$ an open $F_{\sigma}$ set with $A \cap S=\varnothing$. We must show closure $(A) \cap S$ $=\varnothing$. Since $X$ is compact and totally disconnected, $A=\cup A_{n}$, where the $A_{n}$ are clopen sets. If $f_{n}$ is the characteristic function of $A_{1} \cup \cdots \cup A_{n}$, then $0=\int f_{n} d m$ for all $n$. If $f$ is the characteristic function of the clopen set closure $(A)$, then $f=\bigvee f_{n}$, whence $0=\int f d m$, and so closure $(A) \cap S=\varnothing$.) Thus the support of each $m$ in $P\left(T^{*}\right) \cap \Sigma(X)$ in the hypothesis of Theorem 1.1 is a $P$-set. Let $K=$ closure $\cup\left\{\sup (m): m\right.$ in $\left.P\left(T^{*}\right)\right\}$. Since $P\left(T^{*}\right) \cap \Sigma(X)$ is weak-* dense in $P\left(T^{*}\right)$, we have

$$
K=\operatorname{closure}\left(\cup\left\{\sup (m): m \text { in } P\left(T^{*}\right) \cap \Sigma(X)\right\}\right) .
$$

Since $X$ is quasi-stonian and each set in the union is a $P$-set, it follows from [V, Theorem 2] that $K$ is also a $P$-set. (Again, we sketch a proof. Let $K=$ closure $\left(\cup K_{a}\right)$, where the $K_{a}$ are $P$-sets, and $A$ an open $F_{\sigma}$ with $A \cap K=\varnothing$. Suppose closure $(A) \cap K \neq \varnothing$. Since closure $(A)$ is open, it follows that closure $(A)$ $\cap K_{a} \neq \varnothing$ for some index $a$. But $A \cap K_{a}=\varnothing$ and $K_{a}$ is a $P$-set, so we have a contradiction.)

Now $K$ is a $T$-invariant set, i.e., $f \mid K=0$ implies $T f \mid K=0$ [S, Theorems 1.1, 1.3]. Hence $T$ induces in a natural way an operator $T_{0}$ on $C(K)$. Namely if $g$ is in 
$C(K)$ and $x$ is in $K$, let $\bar{g}$ in $C(X)$ be an extension of $g$, and let $T_{0} g(x)=T \bar{g}(x)=$ $\int \bar{g} d\left(T^{*} \delta_{x}\right)$. Since $K$ is a $P$-set, [A $\mathbf{A}_{2}$, Proposition 3.5] implies that if $T_{0}$ is strongly ergodic, then so is $T$. Thus it suffices to prove that the restricted operator $T_{0}$ is strongly ergodic.

Imitating a construction of Gordon [G], let

$$
Y=\bigcup\left\{\sup (m): m \text { in } P\left(T^{*}\right) \cap \Sigma(X)\right\} .
$$

We denote the original topology of $X$ (and hence of $Y$ and closure $(Y)=K$ ) by the name $\alpha$. We shall show later that if $f$ is in $C(X)$, then $T f_{n} \mid Y$ converges uniformly on $Y$, hence on $K=\operatorname{closure}(Y)$.

Following [G, Section 5], we introduce a new topology $\delta$ on $Y$, having as base of open sets all sets of the form $X_{m}=\sup (m)$, where $m$ is any $\sigma$-additive measure whose support is contained in $Y$. We show that $X_{m} \cap X_{p}=X_{q}$ for some $q$ in $\Sigma(X)$ as follows: since $X_{m}$ is a $P$-set and $X_{p}$ a support set, $X_{m} \cap X_{p}$ is $\alpha$-clopen in $X_{p}$. If $f$ is the characteristic function of $X_{m} \cap X_{p}$, put $d q=f d p$ [ $\mathbf{A}_{1}$, Theorem 1]. Clearly $q$ is $\sigma$-additive. Moreover since $X_{m} \cap X_{p}$ is $\alpha$-open in $X_{p}$ for each $X_{m}, \delta$ is a weaker topology on $X_{p}$ than $\alpha$ is. Since $\delta$ is Hausdorff and $\left(X_{p}, \alpha\right)$ is compact, $\left(X_{p}, \delta\right)$ is compact and $\alpha=\delta$ on $X_{p}$. Thus, each $X_{p}$ is open and compact in the $\delta$-topology. (To show $\delta$ is Hausdorff, let $x \neq y$ in $X_{p}$, and find $A, B$ clopen and disjoint in $\left(X_{p}, \alpha\right)$ such that $x$ is in $A$ and $y$ is in $B$. Then $\chi_{A} d p$ and $\chi_{B} d p$ define members of $\Sigma$ with disjoint supports containing $x$ and $y$ respectively.)

Let $\tilde{Y}$ be the Stone-Čech compactification of $(Y, \delta)$. To show that $\tilde{Y}$ is hyperstonian we depart from Gordon's procedure [G, Section 6] and show (i) $Y$, and hence $\tilde{Y}$, is extremally disconnected, and (ii) the union of the supports of normal measures is dense in $\tilde{Y}$ [Sch, p. 121].

For (i), we shall be using the $\delta$-topology except where otherwise mentioned. Let $V$ be open and let $x$ be in closure $(V)$. We must show $x$ is in interior(closure( $V)$ ). Fix an $X_{m}$ containing $x$. Then $x$ is in $V \cap X_{m}$, which is an open subset of $X_{m}$. If $X_{p}$ is any basic neighborhood of $x, X_{p} \cap\left(V \cap X_{m}\right)$ contains $x$ and is an open subset of $V \cap X_{m}$, and hence $x$ is interior to $V \cap X_{m}$ in the subspace topology of $X_{m}$. But on $X_{m}, \alpha=\delta$, and $X_{m}$ is extremally disconnected for $\alpha$ [Se, Theorem 2.2], so $x$ is interior to the closure in $X_{m}$ of $V \cap X_{m}$. Hence there exists a basic open set $X_{q}$ with $x \in X_{q} \subset X_{m}$ and $X_{q} \subset X_{m}$-closure $\left(V \cap X_{m}\right) \subset Y$-closure $(V)$. Thus, $x$ is interior to closure $(V)$.

For (ii) we show that if $m$ is in $P\left(T^{*}\right) \cap \Sigma(X)$, then with respect to the $\delta$-topology on $\tilde{Y}$ it becomes a normal measure. As noted in $\$ 1.3$, it suffices to show that if $F$ is a $\delta$-closed nowhere dense set, then $m(F)=0$. Let $S=X_{m}$, a $\delta$-clopen set. Since $S$ is open, $F \cap S$ is closed and nowhere dense in the relative $\delta$-topology of $S$; and since the relative $\alpha$ and $\delta$-topologies coincide on $S, F \cap S$ is closed and nowhere dense in the relative $\alpha$-topology of $S$. We now show that there exists in $X$ an $\alpha$-closed $G_{\delta}$ set $W, \alpha$-nowhere dense in $X$, such that $F \cap S \subset W \cap S$. Let $V_{n}$ be $\alpha$-open sets with $F \cap S \subset V_{n}$ and $m(F)=m(F \cap S)=\lim m\left(V_{n}\right)$. Since $F \cap$ $S$ is $\alpha$-closed, hence $\alpha$-compact, we may assume each $V_{n}$ is $\alpha$-clopen, and $Z=$ $\cap V_{n}$ is an $\alpha$-closed $G_{\delta}$ set with $F \cap S \subset Z$ and $m(Z \backslash F)=0$. Now $Z \cap S$ is 
$\alpha$-nowhere dense in $S$. (For otherwise there exists $\alpha$-clopen $B$ with $\varnothing \neq B \cap S \subset$ $Z \cap S$. $B \cap S \nsubseteq F \cap S$, since $F \cap S$ is $\alpha$-nowhere dense in $S$, so $(B \backslash F) \cap S \neq$ $\varnothing$, whence $m(B \backslash F)>0$. But $(B \backslash F) \cap S \subset(Z \backslash F) \cap S$, so $m(Z \backslash F)>0$, a contradiction.) Since $X$ is quasi-stonian, the set $A=\alpha$-interior $(Z)$ is clopen. Since $Z \cap S$ is nowhere dense in $S, A \cap S=\varnothing$, so $W=Z \backslash A$ is a nowhere dense $\alpha$-closed $G_{\delta}$ with $W \cap S=Z \cap S \supset F \cap S$.

Finally we show $m(W)=0$, and hence $m(F)=0$. But $W$ has the form $W=$ $\cap A_{n}$, where the $A_{n}$ are $\alpha$-clopen, $A_{1} \supset A_{2} \supset \ldots$ If $f_{n}$ is the characteristic function of $A_{n}$, then since interior $(W)=\varnothing$, we have $\wedge f_{n}=0$, and so $m(W)<$ $\lim \int f_{n} d m=0$. (ii) is proved.

We now show that our original operator $T$ induces an operator $S$ on $C(\tilde{Y})$. If $m$ is in $P\left(T^{*}\right) \cap \Sigma(Y)$, then for $x$ in $X_{m}$ we have $\sup \left(T^{*} \delta_{x}\right) \subset X_{m}$. If $f$ is a $\delta$-continuous function on $\tilde{Y}$ and $x$ in $X_{m}, f \mid X_{m}$ is $\alpha$-continuous on $X_{m}$, and we define $S f(x)=\int f d\left(T^{*} \delta_{x}\right)$. Then $S f$ is defined on all $\tilde{Y}$ as the Stone-Čech extension.

We complete the proof by showing that $S$ is uniformly ergodic, whence, if $f$ is in $C(K)$, then $T_{n} f \mid Y$ converges uniformly, and hence so does $T_{n} f \mid K$. To apply Proposition 2.2, we must prove $P\left(S^{*}\right) \cap \Sigma_{0}(Y)$ is weak-* dense in $P\left(S^{*}\right)$. (Note that the $\delta$-topology introduces no new regular Borel measures on $Y$. For if $m$ is one whose support is contained in $Y$, then since each $X_{p}$ is a $\delta$-clopen set, $m\left(X_{p}\right)>0$ whenever $\sup (m) \cap X_{p} \neq \varnothing$. Hence support $(m)$ is contained in a countable collection of the $X_{p}$ sets. The $\alpha$-closure of the union of this collection is contained in $Y$, and is also an $X_{p}$ set: if $X_{p(i)}$ are the sets in question, just let $p=\Sigma 2^{-i} p(i)$.)

Suppose $P\left(S^{*}\right) \cap \Sigma_{0}(Y)$ is not weak-* dense in $P\left(S^{*}\right)$, and let $m_{0}$ be in $P\left(S^{*}\right)$, but not in *-closure $\left(P\left(S^{*}\right) \cap \Sigma_{0}(Y)\right)$. By separation [D, p. 22], there exists $f$ in $C(Y)$ such that $p(f)<-1$ for all $p$ in $P\left(S^{*}\right) \cap \Sigma_{0}(Y)$, and $m_{0}(f)>0$. Now $\left\{S_{n} f\right.$ : $n>1\}$ is a norm bounded set in $C(Y)$, and since $C(\tilde{Y})$ is the dual of $\Sigma_{0}(Y)$, it is precompact. Let $g$ in $C(Y)$ be a $\sigma\left(\Sigma_{0}(Y), C(\tilde{Y})\right)$ cluster point, and let $\left\{S_{n(i)}: i\right.$ in $\left.I\right\}$ be a subnet such that $\int S_{n(i)} f d p \rightarrow \int g d p$ for all $p$ in $\Sigma_{0}(Y)$. Hence $\int g d m_{0}>0$ and $\int g d p<-1$ for all $p$ in $\Sigma_{0}(Y)$. Further, $S g=g$. (Proof: $\left\|S_{n(i)} S g-S_{n(i)} g\right\|<$ $n(i)^{-1}\left\|S^{n(i)} g-g\right\|<2 n(i)^{-1}\|g\| \rightarrow 0$, whence $\int S g d p=\int g d p$ for all $p$ in $\Sigma_{0}(Y)$. Since, as noted above, $\Sigma_{0}(\tilde{Y})$ is weak-* dense in $C(\tilde{Y})^{*}$, we have $S g=g$.) If $p$ is an extreme point of $P\left(S^{*}\right)$, then $g$ is constant on $\sup (p)$ [S $S_{1}$ Theorem 1.11]. If, further, $\sup (p) \subset X_{m}$ for some $m$ in $P\left(S^{*}\right) \cap \Sigma_{0}(Y)$, then since by hypothesis $P\left(T^{*}\right) \cap$ $\Sigma(Y)$ is dense in $P\left(T^{*}\right)$ and the $\alpha$-topology on $X_{m}$ is the same as the $\delta$-topology, it follows that $p(g) \leqslant-1$ and hence $g \leqslant-1$ on $\sup (p)$. By Krein-Milman, $m$ is in the weak-* closed convex hull of the extreme points of $P\left(S^{*}\right)$, and it is elementary to show that these extreme points may be assumed to have supports contained in $\sup (m)=X_{m}$. Hence

$$
X_{m}=\operatorname{closure}\left(\cup\left\{\sup (p): p \text { extreme, } \sup (p) \subset X_{m}\right\}\right),
$$

whence $g \leqslant-1$ on $X_{m}$. It follows that $g<-1$ on $Y$, and, by density, on $\tilde{Y}$. But this contradicts $m_{0}(g) \geqslant 0$. This proves $P\left(S^{*}\right) \cap \Sigma_{0}(Y)$ is *-dense in $P\left(S^{*}\right)$, and we are through. 
3.2. Proof of the corollary.

(a) implies (b). If (a), then the theorem implies that $T$ is strongly ergodic, and we must show that $\operatorname{dim} F(T)<\infty$. Since by $\left[S_{1}\right.$, Theorem 1.11$], \operatorname{dim} F(T)<$ number of extreme points in $P\left(T^{*}\right)$, it suffices to prove that the latter set is finite. Suppose $m_{1}, m_{2}, \ldots$ are distinct extreme points, and let $S_{i}=\sup \left(m_{i}\right)$. Since $T$ is strongly ergodic, the sets $S_{i}$ are pairwise disjoint. (By [ $\mathbf{S}_{1}$, Theorem 2.7], invariant functions separate the $m_{i}$, and by [ $S_{1}$, Theorem 1.11], an invariant function is constant on each $S_{i}$.) Since $P\left(T^{*}\right) \subset \Sigma(X)$, the $S_{i}$ are $P$-sets, and hence a routine induction gives a pairwise disjoint sequence of clopen sets $A_{i}$ with $S_{i} \subset A_{i}$. Define $f_{i}$ in $C(X)$ to be the characteristic function of $B_{i}=$ closure $\cup_{n>i} A_{n}$. Then $\wedge f_{n}=0$, while if $m$ is a weak-* cluster point of $\left\{m_{i}\right\}$, then $\int f_{n} d m=1$ for all $n$. Thus $m$ is an element of $P\left(T^{*}\right)$ which is not $\sigma$-additive, contrary to hypothesis.

(b) implies (a). This is the easy part; see [An].

ADDED IN PROOF. In his thesis, D. Axmann gives a treatment of Ando's condition in the context of vector lattices, and shows that under this condition $T$ is actually ergodic in the uniform operator topology (Struktur und Ergodentheorie irreduzibler operatoren auf Banachverbänden, Dissertation, Eberhard-Karls-Universität zu Tübingen, 1980, Chapter 4). I do not know whether our condition implies uniform ergodicity.

\section{REFERENCES}

[An] T. Ando, Positive Kontraktionen in $C(X)$, Studia Math. 31 (1968), 173-187.

[A] R. Atalla, Measure theoretic behavior of closed sets, Comm. Math. Univ. Carolinae 19 (1978), 697-703.

[A $\left.\mathrm{A}_{2}\right] \ldots$, Generalized almost-convergence vs. matrix summability, Colloq. Math. (to appear).

[D] M. Day, Normed linear spaces, 1st ed., Springer, Berlin and New York, 1962.

[G] H. Gordon, The maximal ideal space of a ring of measurable functions, Amer. J. Math. 88 (1966), 827-843.

[ $\left.\mathrm{U}_{1}\right]$ S. Lloyd, On the mean ergodic theorem of Sine, Proc. Amer. Math. Soc. 56 (1976), 121-126.

$\left[\mathrm{u}_{2}\right] \ldots$, Two lifting theorems, Proc. Amer. Math. Soc. 42 (1974), 128-134.

[Sa] R. Sato, Invariant measures and ergodic theorems for positive operators on $C(X)$ with $X$ quasi-stonian, Math. J. Okayama Univ. 22 (1979), 77-90.

[Sch] H. Schaefer, Banach lattices and positive operators, Springer, Berlin and New York, 1974.

[Se] G. Seever, Measures on F-spaces, Trans. Amer. Math. Soc. 133 (1968), 267-280.

[S $\mathrm{S}_{1}$ R. Sine, Geometric theory of a single Markov operator, Pacific J. Math. 27 (1968), 155-166.

$\left[\mathrm{S}_{2}\right] \ldots$, A mean ergodic theorem, Proc. Amer. Math. Soc. 24 (1970), 438-439.

[V] A. Veksler, P-sets in topological spaces, Dokl. Akad. Nauk SSSR 193 (1970) = Soviet Math. Dokl. 11 (1970), 953-956.

Department of Mathematics, Ohio Universtty, Athens, Oho 45701 